\title{
Effect of Regular Physical Exercise on the Progression of Erectile Dysfunction Among Male Patients with Diabetes Mellitus
}

\author{
Anees Alyafei \\ Preventive Medicine, Primary Health Care Corporation, Doha, Qatar \\ Email address: \\ qat900@yahoo.com \\ To cite this article: \\ Anees Alyafei. Effect of Regular Physical Exercise on the Progression of Erectile Dysfunction Among Male Patients with Diabetes Mellitus. \\ Clinical Medicine Research. Vol. 9, No. 4, 2020, pp. 74-84. doi: 10.11648/j.cmr.20200904.12
}

Received: July 8, 2020; Accepted: July 20, 2020; Published: July 30, 2020

\begin{abstract}
There is no agreed management for Erectile Dysfunction (ED) for male patients with Diabetes Mellitus (DM). Regular Physical Exercise (PE) starts to be a more exciting area that needs further focus. This review attempts to gather available data about the ED burden, etiology, risk factors, assessment, and management. Further, it highlights the available evidence on the effect of PE on the progression of ED and the possible underline mechanisms. The available evidence is hugely supportive of the beneficial role of PE in the ED. The cardiovascular, neurological, endothelial, metabolic, and overall health benefits of PE are repeated mechanisms reported by many researchers. The possible explanation is quite not clear due to the complexity and bidirectional effect on many aspects related to the changes during PE. Further interventional studies are needed to determine the superiority of each factor. All physicians managing DM and ED are strongly invited to involve PE as part of their management plan in conjunction with other available treatment options. Research has to look after which type, duration, frequency, and intensity of PE is sufficient for detectable improvement in ED.
\end{abstract}

Keywords: Diabetes Mellitus, Erectile Dysfunction, Physical Exercise, Male

\section{Introduction}

Male Erectile Dysfunction (ED) is a well-recognized complication of Diabetes Mellitus (DM). Historically, it was underestimated as a core long term diabetic complication. The widespread global knowledge and advances in pharmacological glycemic control management make ED of great interest for many researchers. Medical literature frequently highlighted the effect of Physical Exercise (PE) over metabolic control, and body components on diabetics but not much on the progression of ED. This review aims to summarize ED from epidemiological, pathological, clinical angel comprehensively. Also, it will address the available evidence and possible physiological mechanisms on the effect of regular PE on the ED progression as a remarkably effective modality of management.

\section{Definition}

There is frequent misuse of two fundamental terms of ER and impotence globally. Erectile Dysfunction is defined as the persistent failure to sustain a penile erection of adequate rigidity for sexual intercourse. $[1,2]$ On the other hand, impotence is reserved for those males who suffer erectile failure for the period of intercourse most of the time or (75\%) of the time. Currently, ER is the recognized term used since (1992), when the National Institutes of Health (NIH) Consensus Development Conference recommended it. [3]

\section{Epidemiology}

\subsection{Prevalence}

The prevalence of ED is varied considerably in the general population and increasing progressively in a more linear manner with age where it is as low as (2.0-9.0\%) for those younger than (40) years. $[4,5]$ While it can reach as much as $(10.0 \%-71.0 \%)$ among the elderly age group. [6-8] Erectile dysfunction is as common as three folds in DM patients in comparison to general population. $[9,10]$ Among male DM patients the prevalence is 
markedly higher with noticeable variability between $(57.0 \%$ $75.0 \%$ ). Such prevalence among male DM patients can be greatly exponentially increase if other comorbidity coexists especially Cardiovascular Disease (CVD). [11]

\subsection{Etiology}

Erectile dysfunction causes are well recognized. Causes basically can be stratified into three main groups; organic, psychologic and medication induced. Each group is further subdivided into different subcategories. Summary of all ED causes are shown in Table 1. But more important to recognize the risk factors related to DM patients.

\subsection{Risk Factors}

The increased probability of getting any sort of ED with progression in age is not negotiable. Where, many references frequently confirmed this fact not only for male DM patients but as well as for the general population. [12] Accumulative evidence clearly addressed the following risk factors to increase the occurrence of ED:

1) Diabetes Mellitus duration: there is a positive linear relation between DM duration and ED prevalence.

2) Presence CVD risk factors: it also includes coronary heart diseases, hypertension, strokes, and as well as peripheral vascular diseases and dyslipidemia. Increasing the CVD risk among DM patients is almost four times more than the healthy population. [13-15] Additionally, many authors reported that ED is a valid early marker for presence of CVD. [16-18]

3) Obesity: with an increase in Body Mass Index (BMI), the risk of ED is increasing. Further evidence that supports the relation of high BMI and ED is the reverse benefit of losing weight and marked improvement in ED among male DM patients. [19]

4) Sedentary Lifestyle: is significantly linked to ED owing to indirect relation to the BMI, physical fitness, and its relation to glycemic control and CVD prevalence. [20]

5) Metabolic Syndrome: is defined as the co-occurrence of several CVD risk factors, obesity, including insulin resistance, dyslipidemia, and hypertension. [21] The complex interactions between all related risk factors of metabolic syndrome are explanatory concerning ED. Furthermore, ED can be a predictor factor for the development of metabolic syndrome, particularly among obese patients. [22, 23]

6) Lower urinary tract disease and benign prostatic hyperplasia are associated with ED. Peer-reviewed data suggested this association. However, many authors recommended the testing of both urinary tract symptoms \& prostatic hyperplasia and ER when a male patient presents with one of them. [24]

7) DM associated complications e.g. retinopathy \& neuropathy aggravate or accelerate ED in male DM patients. [25]

8) Depression: is bidirectional related to $\mathrm{ED}$ in the literature. It could be a risk factor or an outcome for ED. [26, 27]

\section{Pathophysiology}

The exact pathophysiology of ED in male DM patients is still obscured. Although, the accumulative knowledge supports the collective synergistic interaction between psychological, central $\&$ autonomic neuropathy, endothelial dysfunction, and smooth muscle dysfunctions. Such disturbances are quite common among DM patients. [28] Many authors reported the effect of raised Advanced Glycation End-products (AGEs) and elevated oxygen free radicals, impaired Nitric Oxide (NO) synthesis, upregulated RhoA/Rho-kinase pathway, amplified endothelin $\beta$ receptor binding sites \& ultrastructural changes, NO-dependent selective nitrergic nerve degeneration and impaired cyclic guanosine monophosphate (cGMP)-dependent kinase-1. [29-31]

\section{Evaluation}

Erectile dysfunction can be diagnosed through detailed history taking and clinical examination. Further, any underlying etiology must be considered and managed. There are many validated tools to assess ED. International Index of Erectile Function (IIEF) and the Sexual Health Inventory for Men (SHIM) are among the commonest readily wide clinical tools. [32-35]

Table 1. Organic, Psychologic and Medication Induced Causes of Erectile Dysfunction.

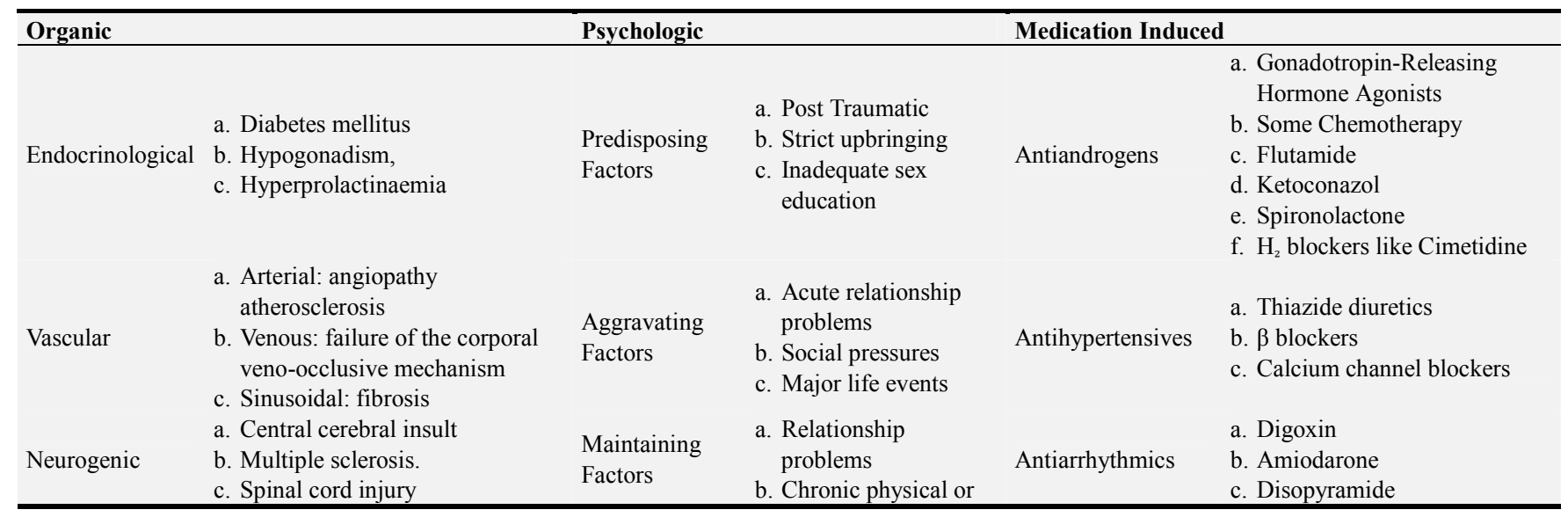




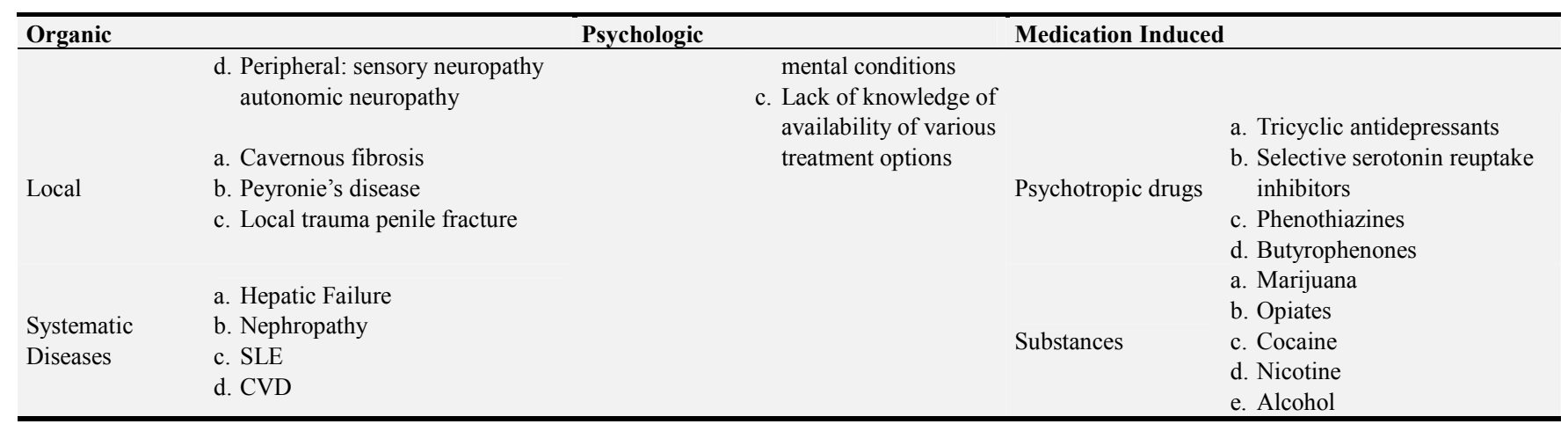

SLE Systemic Lupus Erythematosus, CVD Cardiovascular Disease

\section{Management}

The usual management has four main routs very often more than one routs being used. Table 2 outlines the major practiced management for ED. [36]

1. Psychological Support including psychosexual \& partner therapy, cognitive behavioral therapy, and management of related anxiety or other psychological co-existing condition.

2. Healthy Lifestyle Modification including diet regimen, physical exercise program, weight management, sleep hygiene advice, breaking bad habits like sedentary lifestyle and stress coping. Further, management for smoking cessation, drug abuse, alcohol, and other addiction related materials must be carried out.

3. Pharmacotherapy:

a. Use of oral Phosphodiesterase type 5 inhibitors (PDEsI5) e.g. Sildenafil or Viagra, Tadalafil, and Vardenafil.

b. Testosterone supplement therapy is preserved for low serum concentration of testosterone conditions like in case of hypogonadism. c. Often combination of PDEs-I5 and testosterone prescribed for ED among elderly age group with confirmed low serum testosterone. [37-39]

4. Invasive Management:

a. Intracavernosal injection where usually the patient injects his penis with drugs. Frequently prescribed medications are Alprostadil (prostaglandin E1), Papaverine, and Phentolamine. The effect is fast on most of the patients but usually is associated with side effect of priapism and penis fibrosis if no prober education to the patients. $[40,41]$

b. Vacuum constrictive device, where a negative pressure applying device is used on the penis shaft. It basically draws more blood to corpora cavernosa which ease the erection. Usually such modality is for patients failed on other management options. [42]

c. Penile prosthesis where implants surgery done as the last option if the above-mentioned options failed.

d. Combination therapy, where more than one management option combined together.

Table 2. Management Outline of Erectile Dysfunction.

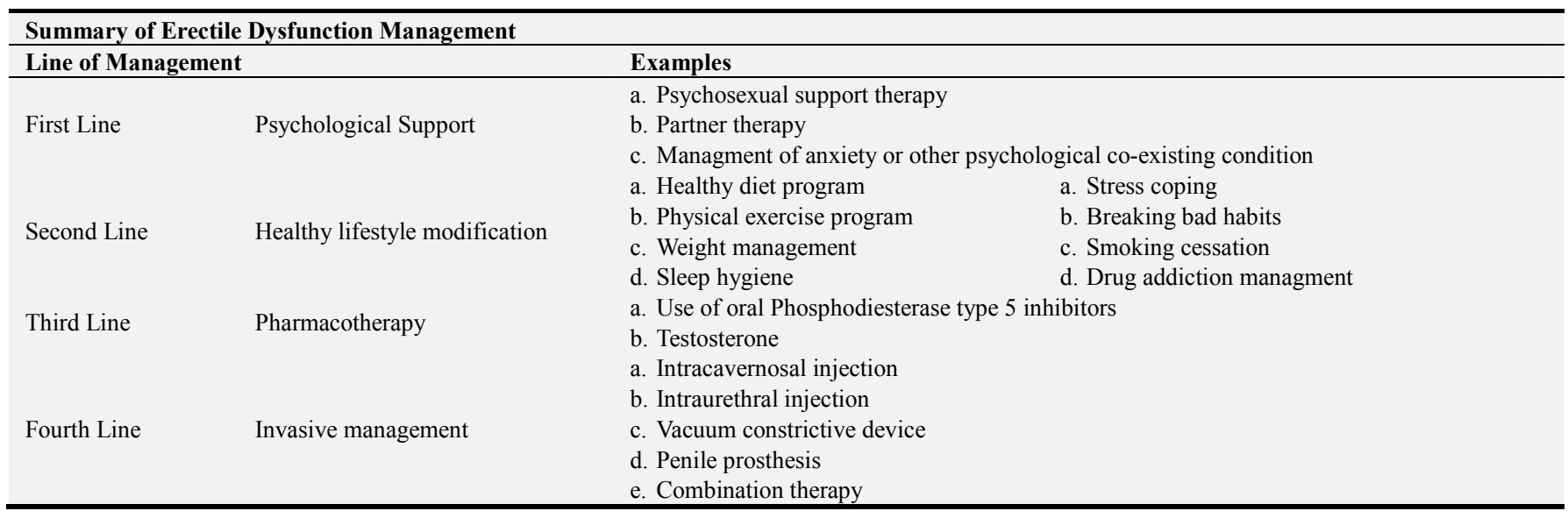

\section{Evidence on the Effect of Exercise on Erectile Dysfunction}

Most of DM male patients show a satisfactory response to the usual pharmacological ED management. However, evidence declares the gradually reduced effect with time, the evidence is currently supporting the fact that healthy lifestyle modifications are associated with reduced ED among males with DM. [43, 44] One essential component of healthy lifestyle modification is physical exercise. 
Physical exercise is defined as a structured, repetitive movement of groups of muscles to obtain health-related or/ and skill-related benefits. Health-related benefits mainly include cardiopulmonary fitness, body components improvement, muscular strength \& endurance, and flexibility. While skilled related benefits are coordination, reaction time, balance, agility, power, and speed. $[45,46]$

Available data about the relation between PE and DM focuses on the biochemical changes related to the glycemic control rather than a clinical improvement on the DM complications. Literature evidence is progressively stressing the need for PE as a very cost-effective modality for the management of ED as well as DM simultaneously. Many authors found the idea of making healthy lifestyle modifications, mainly with the PE program, extremely attractive to combine it with other ED management options.

It is essential to clarify the definition of PE as it is usually misused with physical activity. Physical activity is a very general term that describes any movement that utilizes energy more than resting energy. Physical activity is not repetitive and has nothing to do with health or individual physical skills. While PE is defined as repeated structured movements of muscle or group of muscles to reach healthrelated and or skilled related benefits. [47]

The health-related benefits are improved body composition (e.g., muscle mass, fat mass, weight, and waist circumference), cardiopulmonary fitness (e.g., reduction in resting heart rate, increase in $\mathrm{VO} 2$ max, and improvement in lung volumes), muscle strength, and, flexibility. The skillsrelated benefits are balance, agility, coordination, reaction time, power, and speed. [46]

Generally, PE can be of two kinds aerobic and resistance exercises. The former is those exercises that raise the heart rate and energy expenditure. While resistance exercise is moving a group of muscles against weight, which could be body or object weight. [48] The best examples of aerobic exercises are cycling, swimming, and running. The best example of resistance exercises are dumbbells lifting and pushups.

For diabetic patients, it is recommended by the American College of Sports Medicine to exercise a total of (300) minutes of moderate-intensity aerobic exercise per week, which is equivalent to 60 minutes per session, five times a week. Furthermore, two to three sessions in non-consecutive days of resistance exercise of (60) minutes each per week significantly impact the general health of people with diabetes. Most recommendations include having mixed $\mathrm{PE}$ programs of both aerobic and resistance exercises. Three to five days for aerobic exercises and tow non-consecutive days for resistance exercises are enough to produce health effects. [49]

For maximum benefits from the PE, all diabetic patients have to get a medical clearance or certificate from a physician before starting any PE program. American College of Sports Medicine before commencing $\mathrm{PE}$, it is fundamentally vital to test random blood sugar, which should not be less than $(90 \mathrm{mg} / \mathrm{dl})$ to start the exercise, ingestion of (15-30) grams of sugar is a must. If random blood sugar is between $(90-250 \mathrm{mg} / \mathrm{dl})$, it is safe to perform PE, unlike if it is more than $(250 \mathrm{mg} / \mathrm{dl})$ where ketone tests, not starting the $\mathrm{PE}$, and consulting the treating physician are recommended. Moreover, a gradual warming up and a slow cooling down are particularly essential to prevent muscle cramps and glucose hypoglycemia. After the exercise session, drink enough water and resume regular eating and DM management regimen as prescribed by the treating physician. [50]

In (2000), Feldman HA et al. reported the lower probability of ED among more physically active participants who spent ( $>200 \mathrm{kcal} /$ day) of regular PE in comparison with $(<200 \mathrm{kcal} / \mathrm{day})$. The same study, which is part of the Massachusetts Male Aging Study, supported the fact of reduced ED with a reduction in both smoking and obesity. [12] This could be explained by the fact that more than (200 $\mathrm{Kcal} /$ day) is sufficient to enhance both cardiovascular and neuropathy related factors such as improvement of the peripheral circulation and innervation. Similar findings also documented from Derby et al. regarding the effect of PE even if started in midlife, which is capable of reversing changes related to ED. [20] Also, Van Kampen M et al. found noticeable improvement while assessing the effect of PE on (51) patients with ED. More than two-thirds of them (70.6\%) showed regaining or improvement in their erection following pelvic floor exercise program of one session a week for four months. This could be related to improvement of the localized blood circulation in the pelvic area, which is usually of lesser importance area for PE. [51]

Bacon CG and his team confirmed the same findings on a bigger cohort of men with ED during (2003). Where there was an inverse association between PE and ED. Those with more weekly vigorous PE showed (30\%) lower risk of ED in comparison with those with no PE. [14] This is explained by the dose related effect which is inversely proportionate with all risk factors as well as DM complications. Furthermore, the chronic benefits of the PE contribute to such outcome of Bacon study.

Nicolosi A et al. addressed similar findings from a bigger cross-sectional multi-countries study. It included (40-70) years old males from Brazil, Italy, Japan, and Malaysia (1997-1998). Where negative association was established between regular PE and the incidence of ED. [52] Lamina Sikiru et al. came with the equivalent results via systematic review with a metanalysis study for five clinical trials that measured the ED improvement by IIEF. The mean IIEF improved significantly from (13.91) in the pre-exercise stage to (16.74) following the EP interventions on (385) participants with hypertension. [53]

There are similar findings elsewhere titled the importance and effect of the regular PE in the progression of ED among males with chronic diseases. [43, 54-56] Besides, some authors further support the effect of PE with PDEs-I5 in improving ED than using PDEs-I5 alone. [57] This fact is almost consistent with other findings concerning the synergistic effect of exercise on many pharmacological interventions. It could be due to exercise overall vascular and nerve function effect. 
On the contrary, some other studies showed mild supportive evidence on the effect of PE on ED and called for more clinical trials. Wing and his team found worsening or no change in the ED on the majority of the participants. He conducted a one-year trial to assess the ED on diabetic patients with obesity in Action for Health in Diabetes (AHEAD). A sample of (187) patients showed only (22\%) ED improvement following intensive lifestyle changes with scheduled sessions for weight management and PE. Relatively the sample age was (60) years with comorbidities other than DM, including heart conditions, hypertension, and others. This could partially explain the difference in the results comparing to the references mentioned above. [58] Other population-based and multi-countries studies also illustrated moderate inversely effect of the regular PE on the ED. These studies agreed on the age as an extremely critical risk factor on the response to PE. [59-63]

Further, in the literature, few studies failed to demonstrate the strong positive impact of regular PE on the Cheng ED. JYW et al. reported a negative association through metaanalyses the effect of PE on ED. Further, he and his team proved a simple dose-response relationship between PE and ED. The explanation is not clear, but it could be due to the following factors: Confounding like age, statistical adjustment for different number of variables, definitions of
ED and physical activity, and weak causal inference especially with cross-sectional studies. Nevertheless, also, PE showed low risk on those with vigorous exercise comparing to moderate ones. [64]

\subsection{Mechanism of Physical Exercise Effect on Erectile Dysfunction}

The effect of regular PE on different body systems is well documented. To explain the underlying mechanism that improves the $\mathrm{ED}$ by $\mathrm{PE}$ is quite complicated due to the complexity of the pathophysiology related to the development of ED among male patients with DM. The mechanism looks to be more bidirectional, synergistic on the origin, and of dose-response character. [65]

There is accumulated evidence on the improvement in ED due to cardiovascular, neurological, metabolic, and other associated factors. It is a synergistic and dose-effect role of the PE on the ED, particularly in young and early elderly age group. Furthermore, the significant effect of PE on the ED is extended to improve the mental \& psychological and quality of life of male patients with DM, which both are having a massive influence on the progression of ED. Figure 1 summarizes the most important factors influencing the effect of PE on ED.

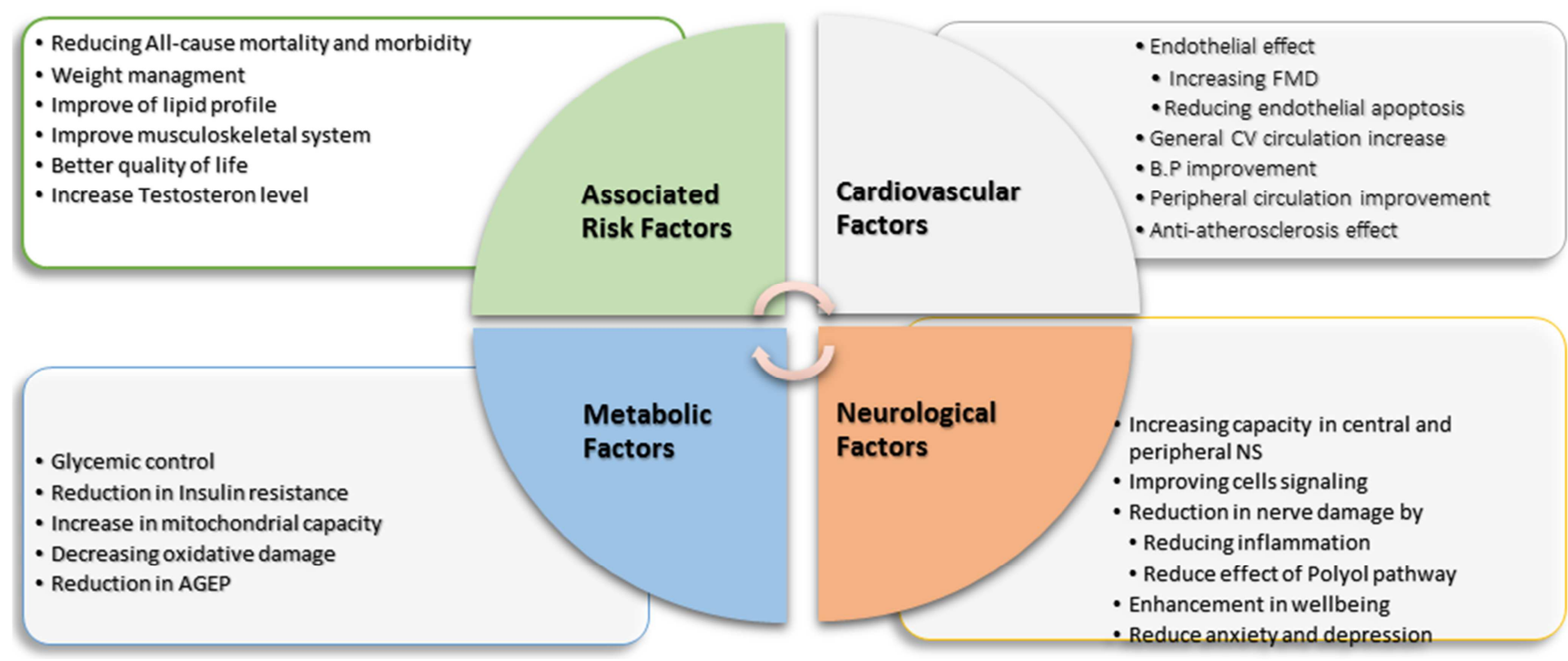

FMD Flow-Mediated Dilation, CV Cardiovascular, B. P Blood Pressure, NS nervous system, AGED Advanced Glycated End Products

Figure 1. Mechanisms of regular physical exercise that improves the erectile dysfunction.

\subsubsection{Effect of Physical Exercise on Cardiovascular Factors}

The evidence of the effect of DM on cardiovascular system is well known. It has multifactorial with synergistic effect. Patients with DM are at four folds increase in the CVD risk. [15] Physical exercise is well known to increase the heart rate, cardiac output, systolic blood pressure, and peripheral perfusion to meet the exercise's metabolic and energy needs. $[66,67]$ Moreover, exercise is a potent stimulant for autonomic regulation on the overall cardiac function. This leads to catecholamines secretion and subsequent increases in cardiac function, coronary vessel dilatation, and increases on NO bio viability. [68, 69]

Cardiac functions quickly are adapting PE changes from metabolic perspectives by enhancement of metabolic flexibility, energy expenditure, both fatty acid oxidation \& glucose metabolism, insulin sensitivity, mitochondrial biogenesis, antioxidants effect, and the cellular action of the catecholamines. [70, 71] In the long run, PE all related vital signs improve, such as a reduction in the rest heart rate due to the relative physiological cardiac hypertrophy, reduction in the systolic and diastolic blood pressure, and enrichment of 
the peripheral circulation including vessels supplying the penis. [72, 73] Effect of $\mathrm{PE}$ on cardiovascular factors that improve ED comes from enhancement on endothelial function, cardiovascular circulation, including both blood pressure and peripheral circulation.

\subsubsection{Endothelial Effect}

There is some evidence of considerable improvement in the endothelial function following regular PE, where documented enhancement was seen by Flow-Mediated Dilation (FMD) of the brachial artery in the group submitted to PE for eight weeks. [74] Further, the evidence supports the reduction in the endothelial apoptosis as a probable reason to explain the improvement in the endothelial function following regular PE. Also, these changes further supported by a reduction in the inflammatory status associated with DM. [75, 76]

Some authors also included the mechanical shear forces as suggested mechanism. The exerted shear forces on the luminal surface of the endothelial monolayer increases in blood flow and results in boosting the vasodilatory capacity of the arteries and stimulation of NO pathway. [77] The effect of NO is not exclusive for improving the vascular tone but also of anti-atherosclerosis effect by stopping platelets and inflammatory cells from adhering to the vascular surface. [87]

The overall result of improved endothelial functions after regular PE, could be explained by increase amount of (NO), improvement in antioxidant \& immune response, a decrease of plaque volume, a decline in vascular wall viscosity and also a rise in diastolic coronary perfusion. [79]

Additionally, many molecules like Beclin1 and Autophagy-Related Gene 3 (Atg3) increases versus reduction in p62 a classical receptor for autophagy, which boosted autophagy regulation on vessel's endothelium. [80-82]

\subsubsection{Cardiovascular Circulation}

The PE is a vigorous stimulation of catecholamine production in dose and intensity related style. Over releasing of catecholamines i.e., noradrenaline, adrenaline, and dopamine, plays an essential regulatory action on cardiovascular circulation. [83]

Physical exercise on acute phase cause catecholamines to increase heart rate and systolic blood pressure besides other systematic effects. The sensible explanation for long term effects of PE in DM patients are the followings: [84-86]

1) Decrease on resting heart rate is secondary to the relative physiological increase on the left ventricular muscle mass and increase on related cardiac output.

2) Enhancement in the blood pressure is a result to increased sensitivity of aortic baroreceptors, which contributes to the regulation of systolic blood pressure. In addition, diastolic blood pressure is improved due to decrease in the peripheral resistance due to the PE associated vasodilatation effect. Reduced action of the sympathetic nervous system and the renin-angiotensin system were also documented.
There is a parallel improvement in overall lung functions as a response to the demands of Oxygen and cardiac function. All the known lung volumes are increased on patients with DM following eight weeks PE program as studied by Saki et al., Where the diabetics had three sessions a week of moderate intensity exercise of (45-60) minutes length. The improvement in the pulmonary function was due to increase in forced vital capacity and forced expiratory volume. [87] The conjoint improvement in cardiac and pulmonary functions will lead to increase in the $\mathrm{VO}_{2} \max$ and general body fitness. Further to the improvement in the general cardiovascular circulation, PE influence the peripheral circulation as a collective result. All the abovementioned factors of endothelial function, NO pathway, general cardiovascular circulation improve greatly the peripheral and collateral flow to the pelvic area including the penis.

\subsection{Effect of Physical Exercise on Neurological Factors}

The damage effect of DM on the nerve is well documented as a chronic complication. Physical exercise is greatly improving both central and peripheral neurological functions that interfere with the ED more pronounced if combined with phycological therapy. [88, 89] Frequent reports confirmed the change in the peripheral nervous system due to better vascular, neuro signaling, endocrinological functions, as well as the overall nerve health. [90]

$\mathrm{Yu} \mathrm{Gu}$ et $a l$., reported improvement in the nerve functions among patients with DM and prediabetics through recent systematic review done on (2018). Also, he called for further study to assess the optimum dose of exercise that result in the clinical beneficial effect. [91]

Another possible explanation for the improvement in the neurological factor in ED is the holistic reduction in the inflammatory state associated with DM as well as the better control on the metabolic disturbance related to hyperglycemia. Both of these effects will be explained in the next section.

Alternative acceptable explanation for improvement in the neurological factor could be by reducing the negative effect of polyol pathway which is recognized as a cause of tissue damage in DM. Polyol pathway is a metabolic process to convert the glucose into sorbitol and finally fructose, thus also called sorbitol-aldose reductase pathway. [92, 93] Robinson J. documented the effect of regular PE in reducing the negative impact of Polyol pathway in both animal models and human trials. This was critically linked to the improvement of in the re-generation of cutaneous smalldiameter nerve fibers. [94]

It is significant to add the effect of PE on the central nervous system to explain the improvement in ED. Regular PE positively influence wellbeing, high cognitive function, depression or anxiety related to ED, central nervous signaling, balance of various types of neurotransmitters, selfsteam, sleeping pattern and subsequently quality of life and including social life. [95-97] 


\subsection{Effect of Physical Exercise on Metabolic Factors}

Physical exercise's impact on glycemic control is mainly due to a reduction in the overall insulin resistance, besides the whole metabolic regulation on energy utilization. The effect of PE can be divided into acute and chronic. The overall effect is more of a metabolic origin with a more systematic impact with high quality of life improvement. [49]

In the acute effect the following changes are observed following PE:

1) Acceleration in the uptake of glucose into the active skeletal muscles by non-insulin-dependent mechanism.

2) Stimulation of the hepatic gluconeogenesis to meet the need of energy by body systems.

3) Regular PE can improve the instant role of insulin that could be post-exercise till 2-3 days post-exercise session.

4) The risk of getting hypoglycemic effect during or after the PE is minimal unless therapeutic insulin is used.

5) Vigorous exercise may lead to transient hyperglycemia on some diabetic post-exercise.

While for those with daily habit of regular PE the following changes are observed on the long run:

1) Reduction in the probability or deaccelerate occurrence of known long term DM complications. This is related to stabilization of metabolic, glycemic control, improvement of vascular, neurological, and $\mathrm{CV}$ functions.

2) Reduction in all cause of mortality including the CVD.

3) Increasing in overall skeletal muscle mass, with reduce in total body fat mass.

4) Overall great improvement in mental, musculoskeletal, and quality of life.

5) Reduction in the overall DM related inflammatory markers.

It is also interesting to not only emphasis on the hyperglycemia as central point of the ED pathogenesis but to highlight related metabolic changes associated with $\mathrm{PE}$ among DM patients. Further explanation for improvement in ED was suggested by the overall improvement of mitochondrial function. Van Tienen et al. findings which were confirmed also by Phielix Esther and his team, indicated increase in mitochondrial capacity as well as mitochondrial metabolic pathways. [98, 99] The resulted effect of increasing the mitochondrial function is not limited to increasing the muscle anerobic capacity, but it goes to increase in protection of tissue damage by reducing oxidative effect. The regular $\mathrm{PE}$ increases resistance to oxidative damage and thus preventing cellular and tissues damage. [100-103]

Reducing the advanced glycation end products, which are outcomes of exposure to hyperglycemia was also suggested in the literature as possible mechanism for improvement in ED. Advanced glycation end products are considered as biomarkers in tissue damage in DM. The best example is $\mathrm{HbA}_{1} \mathrm{c}$, which is important marker for diagnose and monitor glycemic control. Regular PE helps in the reduction of the overall of advanced glycation end products. [104-108]

\subsection{Effect of the Physical Exercise on Associated Risk Factors}

In addition to the effects mentioned above, PE's additive effect on the progression of ED is supported by a reduction in all other associated risk factors, including obesity, lipid profile, cardiopulmonary capacity, improved musculoskeletal system, mental health, quality of life, and hormonal changes on androgens. [109, 110]

Additional explanation on the effect of regular PE on ED is the better hormonal balance experienced after each PE session on the acute term. One significant effect of PE is the association of increased testosterone after the exercise. This increase is usually reported to be transient and combined with variable elevation of other known androgens. [111, 112]

However, PE's long-term effect on the hormones related to ED is not well consistent in the literature. It was demonstrated to be high while measuring the serum testosterone, as reported by Herbst et al. and confirmed by Kraemer and his team. [113-115] While, Izquierdo et al., concluded no change in serum testosterone after (16) weeks of resistance exercise training for a group of middle-aged men which also documented by Nicklas BJ and his colleagues. [116, 117] The possible explanation could be related to the age of the participants as more proportion of them are more seniors. A more recent controlled clinical trial showed that moderate-intensity aerobic exercise program of (12) months increased dihydrotestosterone and sex hormonebinding globulin with no change on androgens in a group of (102) men aged (40-70) years. [118]

\section{Conclusion}

Erectile dysfunction is a very prevalent complication of DM among males. It is a multifactorial condition that is greatly influenced by age, vascular, endocrinological, and psychological elements. Regular PE is a great cost-effective management option, as reported recurrently by many researchers. Still, further, clinical and interventional studies are needed to assess which component of the PE is more superior to exert the effect on ED. Physicians are sincerely invited to incorporate $\mathrm{PE}$ as part of the management, not exclusively for the seek of ED but to improve the diabetic patients' overall quality of life.

\section{Abbreviation}

AGEs Advanced Glycation End-Products, AHEAD Action for Health in Diabetes, Atg3 Autophagy-Related Gene 3, BMI Body Mass Index, BP Blood Pressure, cGMP cyclic Guanosine Monophosphate, CV Cardiovascular, CVD Cardiovascular Disease, DM Diabetes Mellitus, ED Erectile Dysfunction, IIEF International Index of Erectile Function, NIH National Institutes of Health, NO Nitric Oxide, NS Nervous System, PE Physical Exercise, SHIM Sexual Health Inventory for Men, SLE Systemic Lupus Erythematosus 


\section{Conflict of Interest}

Authors have no financial interest, arrangement, or affiliation with anyone concerning this narrative review that could be perceived as a real or apparent conflict of interest in this study's subject.

\section{Acknowledgements}

We are grateful to those colleagues who took the time and effort to participate in this narrative review.

\section{References}

[1] Chu, Neelima V and Steven V Edelman. "Diabetes and Erectile Dysfunction." Clinical diabetes, vol. 19, no. 1, 2001, pp. 45-47.

[2] Nimesh, Saurabh et al. "Erectile Dysfunction: An Update." Advances in Medical, Dental and Health Sciences, vol. 2, no. $1,2019$.

[3] Consensus, NIH. "Development Panel on Impotence. Nih Consensus Conference. Impotence." Jama, vol. 270, 1993, pp. 83-90.

[4] Braun, Moritz et al. "Epidemiology of Erectile Dysfunction: Results of the "Cologne Male Survey'." International journal of impotence research, vol. 12, no. 6, 2000, pp. 305-311.

[5] Martin-Morales, Antonio et al. "Prevalence and Independent Risk Factors for Erectile Dysfunction in Spain: Results of the Epidemiologia De La Disfuncion Erectil Masculina Study." The Journal of urology, vol. 166, no. 2, 2001, pp. 569-575.

[6] Blanker, Marco H et al. "Erectile and Ejaculatory Dysfunction in a Community-Based Sample of Men 50 to 78 Years Old: Prevalence, Concern, and Relation to Sexual Activity." Urology, vol. 57, no. 4, 2001, pp. 763-768.

[7] Selvin, Elizabeth et al. "Prevalence and Risk Factors for Erectile Dysfunction in the Us." The American journal of medicine, vol. 120, no. 2, 2007, pp. 151-157.

[8] Prins, Jan et al. "Prevalence of Erectile Dysfunction: A Systematic Review of Population-Based Studies." International journal of impotence research, vol. 14, no. 6, 2002, pp. 422-432.

[9] Maiorino, Maria Ida et al. "Diabetes and Sexual Dysfunction: Current Perspectives." Diabetes, metabolic syndrome and obesity: targets and therapy, vol. 7, 2014, p. 95.

[10] Kamenov, ZA. "A Comprehensive Review of Erectile Dysfunction in Men with Diabetes." Experimental and Clinical Endocrinology \& Diabetes, vol. 123, no. 03, 2015, pp. 141-158.

[11] Grover, Steven A et al. "The Prevalence of Erectile Dysfunction in the Primary Care Setting: Importance of Risk Factors for Diabetes and Vascular Disease." Archives of internal medicine, vol. 166, no. 2, 2006, pp. 213-219.

[12] Feldman, Henry A et al. "Erectile Dysfunction and Coronary Risk Factors: Prospective Results from the Massachusetts Male Aging Study." Preventive Medicine, vol. 30, no. 4, 2000, pp. 328-338.
[13] Fung, Maple M et al. "Heart Disease Risk Factors Predict Erectile Dysfunction 25 Years Later: The Rancho Bernardo Study." Journal of the American College of Cardiology, vol. 43, no. 8, 2004, pp. 1405-1411.

[14] Bacon, Constance $G$ et al. "Sexual Function in Men Older Than 50 Years of Age: Results from the Health Professionals Follow-up Study." Annals of internal medicine, vol. 139, no. 3, 2003, pp. 161-168.

[15] Al-yafei, Anees et al. "Assessment of Cardiovascular Disease Risk among Qatari Patients with Type 2 Diabetes Mellitus, Attending Primary Health Care Centers, 2014." The Open Diabetes Journal, vol. 10, no. 1, 2020.

[16] Inman, Brant A et al. "A Population-Based, Longitudinal Study of Erectile Dysfunction and Future Coronary Artery Disease." Mayo Clinic Proceedings, vol. 84, Elsevier, 2009, pp. 108-113.

[17] Chung, Shiu - Dong et al. "Increased Risk of Stroke among Men with Erectile Dysfunction: A Nationwide Population Based Study." The journal of sexual medicine, vol. 8, no. 1, 2011, pp. 240-246.

[18] Dong, Jia-Yi et al. "Erectile Dysfunction and Risk of Cardiovascular Disease: Meta-Analysis of Prospective Cohort Studies." Journal of the American College of Cardiology, vol. 58, no. 13, 2011, pp. 1378-1385.

[19] Blanker, Marco H et al. "Correlates for Erectile and Ejaculatory Dysfunction in Older Dutch Men: A Community-Based Study." Journal of the American Geriatrics Society, vol. 49, no. 4, 2001, pp. 436-442.

[20] Derby, Carol A et al. "Modifiable Risk Factors and Erectile Dysfunction: Can Lifestyle Changes Modify Risk?" Urology, vol. 56, no. 2, 2000, pp. 302-306.

[21] Huang, Paul L. "A Comprehensive Definition for Metabolic Syndrome." Disease models \& mechanisms, vol. 2, no. 5-6, 2009, pp. 231-237.

[22] Demir, Omer et al. "Association between Severity of Lower Urinary Tract Symptoms, Erectile Dysfunction and Metabolic Syndrome." The Aging Male, vol. 12, no. 1, 2009, pp. 29-34.

[23] Kupelian, Varant et al. "Erectile Dysfunction as a Predictor of the Metabolic Syndrome in Aging Men: Results from the Massachusetts Male Aging Study." The Journal of urology, vol. 176 , no. 1,2006 , pp. 222-226.

[24] De Nunzio, Cosimo et al. "Erectile Dysfunction and Lower Urinary Tract Symptoms." European urology focus, vol. 3, no. 4-5, 2017, pp. 352-363.

[25] Zheng, Hui et al. "Predictors for Erectile Dysfunction among Diabetics." Diabetes research and clinical practice, vol. 71, no. 3, 2006, pp. 313-319.

[26] Shabsigh, Ridwan et al. "Sexual Dysfunction and Depression: Etiology, Prevalence, and Treatment." Current urology reports, vol. 2, no. 6, 2001, pp. 463-467.

[27] Seidman, Stuart N and Steven P Roose. "Sexual Dysfunction and Depression." Current psychiatry reports, vol. 3, no. 3, 2001, pp. 202-208.

[28] F Adeniyi, Ade et al. "Diabetes, Sexual Dysfunction and Therapeutic Exercise: A 20 Year Review." Current diabetes reviews, vol. 6 , no. 4, 2010, pp. 201-206. 
[29] Zheng, Hong et al. "Lack of Central Nitric Oxide Triggers Erectile Dysfunction in Diabetes." American Journal of Physiology-Regulatory, Integrative and Comparative Physiology, vol. 292, no. 3, 2007, pp. R1158-R1164.

[30] Moore, Charles R and Run Wang. "Pathophysiology and Treatment of Diabetic Erectile Dysfunction." Asian journal of andrology, vol. 8, no. 6, 2006, pp. 675-684.

[31] Thorve, Vrushali $\mathrm{S}$ et al. "Diabetes-Induced Erectile Dysfunction: Epidemiology, Pathophysiology and Management." Journal of Diabetes and its Complications, vol. 25, no. 2, 2011, pp. 129-136.

[32] Rosen, Raymond $\mathrm{C}$ et al. "Development and Evaluation of an Abridged, 5-Item Version of the International Index of Erectile Function (Iief-5) as a Diagnostic Tool for Erectile Dysfunction." International journal of impotence research, vol. 11 , no. 6,1999 , pp. 319-326.

[33] Rosen, Raymond $\mathrm{C}$ et al. "The International Index of Erectile Function (Iief): A Multidimensional Scale for Assessment of Erectile Dysfunction." Urology, vol. 49, no. 6, 1997, pp. 822-830.

[34] Ghanem, Hussein and Rany Shamloul. "An Evidence-Based Perspective to Commonly Performed Erectile Dysfunction Investigations." The journal of sexual medicine, vol. 5, no. 7, 2008, pp. 1582-1589.

[35] Cappelleri, JC and RC Rosen. "The Sexual Health Inventory for Men (Shim): A 5-Year Review of Research and Clinical Experience." International journal of impotence research, vol. 17 , no. 4, 2005, pp. 307-319.

[36] Shamloul, Rany and Hussein Ghanem. "Erectile Dysfunction." The Lancet, vol. 381, no. 9861, 2013, pp. 153-165.

[37] Jain, Pankaj et al. "Testosterone Supplementation for Erectile Dysfunction: Results of a Meta-Analysis." The Journal of urology, vol. 164, no. 2, 2000, pp. 371-375.

[38] Shabsigh, R et al. "Randomized Study of Testosterone Gel as Adjunctive Therapy to Sildenafil in Hypogonadal Men with Erectile Dysfunction Who Do Not Respond to Sildenafil Alone." Ibid. vol. 179, no. 5, 2008, pp. S97-S102.

[39] Shamloul, Rany et al. "Testosterone Therapy Can Enhance Erectile Function Response to Sildenafil in Patients with Padam: A Pilot Study." The journal of sexual medicine, vol. 2, no. 4, 2005, pp. 559-564.

[40] Hatzimouratidis, Konstantinos and Dimitrios G Hatzichristou. "A Comparative Review of the Options for Treatment of Erectile Dysfunction." Drugs, vol. 65, no. 12, 2005, pp. 1621-1650.

[41] Perimenis, $\mathrm{P}$ et al. "Long - Term Treatment with Intracavernosal Injections in Diabetic Men with Erectile Dysfunction." Asian journal of andrology, vol. 8, no. 2, 2006, pp. 219-224.

[42] Hartmut, P and B Jacques. "Standard Practice in Sexual Medicine." Philadelphia: Blackwell, 2006.

[43] Esposito, Katherine and Dario Giugliano. "Obesity, the Metabolic Syndrome, and Sexual Dysfunction." International journal of impotence research, vol. 17, no. 5, 2005, pp. 391-398.

[44] Penson, David F et al. "Do Impotent Men with Diabetes Have More Severe Erectile Dysfunction and Worse Quality of Life Than the General Population of Impotent Patients?: Results from the Exploratory Comprehensive Evaluation of Erectile
Dysfunction (Exceed) Database." Diabetes care, vol. 26, no. 4, 2003, pp. 1093-1099.

[45] Alyafei, Anees and Wadha Albaker. "Barriers and Facilitators for Regular Physical Exercise among Adult Females Narrative Review 2020." Journal of Community Medicine \& Public Health, 2020.

[46] Medicine, American College of Sports. Acsm's Health-Related Physical Fitness Assessment Manual. Lippincott Williams \& Wilkins, 2013.

[47] Alyafei, Anees "Is Exercise Real Medicine." 2019, doi: 10.13140/RG.2.2.25735.21920.

[48] Colberg, Sheri R et al. "Exercise and Type 2 Diabetes: The American College of Sports Medicine and the American Diabetes Association: Joint Position Statement." Diabetes care, vol. 33, no. 12, 2010, pp. e147-e167.

[49] Caldeira, AO and AJ Leggett. "Erratum Ibid." Ann Phys (NY), vol. 153, 1983, p. 445.

[50] Colberg, Sheri $\mathrm{R}$ et al. "Physical Activity/Exercise and Diabetes: A Position Statement of the American Diabetes Association." Diabetes care, vol. 39, no. 11, 2016, pp. 20652079.

[51] Van Kampen, Marijke et al. "Treatment of Erectile Dysfunction by Perineal Exercise, Electromyographic Biofeedback, and Electrical Stimulation." Physical therapy, vol. 83 , no. 6,2003 , pp. $536-543$.

[52] Nicolosi, Alfredo et al. "A Population Study of the Association between Sexual Function, Sexual Satisfaction and Depressive Symptoms in Men." Journal of affective disorders, vol. 82, no. 2, 2004, pp. 235-243.

[53] Lamina, Sikiru et al. "Therapeutic Effect of an Interval Exercise Training Program in the Management of Erectile Dysfunction in Hypertensive Patients." The Journal of Clinical Hypertension, vol. 11, no. 3, 2009, pp. 125-129.

[54] Kałka, D et al. "Evaluation of Ambulatory Cardiac Rehabilitation Influence on the Intensity of Erectile Dysfunction in Patients with Ischemic Heart Disease." Polski merkuriusz lekarski: organ Polskiego Towarzystwa Lekarskiego, vol. 27, no. 160, 2009, pp. 290-295.

[55] Mak, Rudolf et al. "Prevalence and Correlates of Erectile Dysfunction in a Population-Based Study in Belgium." European urology, vol. 41, no. 2, 2002, pp. 132-138.

[56] Tan, JK et al. "Erectile Dysfunction in Singapore: Prevalence and Its Associated Factors-a Population-Based Study." Singapore Med J, vol. 44, no. 1, 2003, pp. 20-26.

[57] Maio, Giuseppe et al. "Physical Activity and Pde5 Inhibitors in the Treatment of Erectile Dysfunction: Results of a Randomized Controlled Study." The journal of sexual medicine, vol. 7, no. 6, 2010, pp. 2201-2208.

[58] Wing, Rena $\mathrm{R}$ et al. "Effects of Weight Loss Intervention on Erectile Function in Older Men with Type 2 Diabetes in the Look Ahead Trial." Ibid. no. 1, pp. 156-165.

[59] Shaeer, KZM et al. "Prevalence of Erectile Dysfunction and Its Correlates among Men Attending Primary Care Clinics in Three Countries: Pakistan, Egypt, and Nigeria." International journal of impotence research, vol. 15, no. 1, 2003, pp. S8S14. 
[60] Moreira Jr, Eduardo Duarte et al. "Prevalence and Correlates of Erectile Dysfunction in Salvador, Northeastern Brazil: A Population-Based Study." Ibid. vol. 14, no. 2, 2002, pp. S3-S9.

[61] Akkus, Emre et al. "Prevalence and Correlates of Erectile Dysfunction in Turkey: A Population-Based Study." European urology, vol. 41, no. 3, 2002, pp. 298-304.

[62] Nicolosi, Alfredo et al. "Epidemiology of Erectile Dysfunction in Four Countries: Cross-National Study of the Prevalence and Correlates of Erectile Dysfunction." Urology, vol. 61, no. 1, 2003, pp. 201-206.

[63] Cho, BL et al. "Prevalence and Risk Factors for Erectile Dysfuntion in Primary Care: Results of a Korean Study." International journal of impotence research, vol. 15, no. 5, 2003, pp. 323-328.

[64] Cheng, JYW et al. "Physical Activity and Erectile Dysfunction: Meta-Analysis of Population-Based Studies." Ibid. vol. 19, no. 3, 2007, pp. 245-252.

[65] Verboven, Maxim et al. "Effect of Exercise Intervention on Cardiac Function in Type 2 Diabetes Mellitus: A Systematic Review." Sports medicine, vol. 49, no. 2, 2019, pp. $255-268$.

[66] Vega, Rick B et al. "Molecular Mechanisms Underlying Cardiac Adaptation to Exercise." Cell metabolism, vol. 25, no. 5, 2017, pp. 1012-1026.

[67] Olver, T Dylan et al. "Molecular Mechanisms for Exercise Training-Induced Changes in Vascular Structure and Function: Skeletal Muscle, Cardiac Muscle, and the Brain." Progress in Molecular Biology and Translational Science, vol. 135, Elsevier, 2015, pp. 227-257.

[68] Bellavere, $\mathrm{F}$ et al. "Effects of Aerobic or Resistance Exercise Training on Cardiovascular Autonomic Function of Subjects with Type 2 Diabetes: A Pilot Study." Nutrition, Metabolism and Cardiovascular Diseases, vol. 28, no. 3, 2018, pp. 226233 .

[69] $\mathrm{Wu}, \mathrm{Ne} \mathrm{N}$ et al. "Physical Exercise and Selective Autophagy: Benefit and Risk on Cardiovascular Health." Cells, vol. 8, no. 11, 2019, p. 1436.

[70] Thompson, Dylan et al. "Physical Activity and Exercise in the Regulation of Human Adipose Tissue Physiology." Physiological reviews, 2012.

[71] Cassidy, Sophie et al. "High-Intensity Interval Training: A Review of Its Impact on Glucose Control and Cardiometabolic Health." Diabetologia, vol. 60, no. 1, 2017, pp. 7-23.

[72] Ceylan, Asli F et al. "Cardiomyocyte-Specific Knockout of Endothelin Receptor a Attenuates Obesity Cardiomyopathy." Biochimica et Biophysica Acta (BBA)-Molecular Basis of Disease, vol. 1864, no. 10, 2018, pp. 3339-3352.

[73] Wang, Cheng et al. "Aerobic Exercise Interventions Reduce Blood Pressure in Patients after Stroke or Transient Ischaemic Attack: A Systematic Review and Meta-Analysis." British journal of sports medicine, vol. 53, no. 24, 2019, pp. 15151525.

[74] Belardinelli, Romualdo et al. "Effects of Short-Term Moderate Exercise Training on Sexual Function in Male Patients with Chronic Stable Heart Failure." International journal of cardiology, vol. 101, no. 1, 2005, pp. 83-90.
[75] La Vignera, Sandro et al. "Aerobic Physical Activity Improves Endothelial Function in the Middle-Aged Patients with Erectile Dysfunction." The Aging Male, vol. 14, no. 4, 2011, pp. 265-272.

[76] Balducci, Stefano et al. "Anti-Inflammatory Effect of Exercise Training in Subjects with Type 2 Diabetes and the Metabolic Syndrome Is Dependent on Exercise Modalities and Independent of Weight Loss." Nutrition, Metabolism and Cardiovascular Diseases, vol. 20, no. 8, 2010, pp. 608-617.

[77] Hambrecht, Rainer et al. "Effect of Exercise on Coronary Endothelial Function in Patients with Coronary Artery Disease." New England Journal of Medicine, vol. 342, no. 7, 2000, pp. 454-460.

[78] Uematsu, MASAAKI et al. "Regulation of Endothelial Cell Nitric Oxide Synthase Mrna Expression by Shear Stress." American Journal of Physiology-Cell Physiology, vol. 269, no. 6, 1995, pp. C1371-C1378.

[79] Skrypnik, D et al. "Effect of Physical Exercise on Endothelial Function, Indicators of Inflammation and Oxidative Stress." Polski merkuriusz lekarski: organ Polskiego Towarzystwa Lekarskiego, vol. 36, no. 212, 2014, p. 117.

[80] Balligand, Jean-Luc. "Cardiac Salvage by Tweaking with Beta-3-Adrenergic Receptors." Cardiovascular research, vol. 111, no. 2, 2016, pp. 128-133.

[81] Mehanna, Emile et al. "Cardiorespiratory Fitness and Atherosclerosis: Recent Data and Future Directions." Current atherosclerosis reports, vol. 18, no. 5, 2016, p. 26.

[82] Roe, Nathan D and Jun Ren. "Nitric Oxide Synthase Uncoupling: A Therapeutic Target in Cardiovascular Diseases." Vascular pharmacology, vol. 57, no. 5-6, 2012, pp. 168-172.

[83] Kruk, Joanna et al. "Physical Exercise and Catecholamines Response: Benefits and Health Risk: Possible Mechanisms." Free Radical Research, vol. 54, no. 2-3, 2020, pp. 105-125.

[84] Brum, Patricia Chakur et al. "Exercise Training Increases Baroreceptor Gain Sensitivity in Normal and Hypertensive Rats." Hypertension, vol. 36, no. 6, 2000, pp. 1018-1022.

[85] Zinman, Bernard et al. "Physical Activity/Exercise and Diabetes." Diabetes care, vol. 27, 2004, p. S58.

[86] Thomas, Diana et al. "Exercise for Type 2 Diabetes Mellitus." Cochrane Database of Systematic Reviews, no. 3, 2006.

[87] Saki, Hossein et al. "Comparison of the Effects of Aerobic Exercise on Pulmonary Function and Levels of Inflammatory Mediators in Men with Type 2 Diabetes." International Journal of Basic Science in Medicine, vol. 2, no. 2, 2017, pp. 95-100.

[88] van Laake-Geelen, Charlotte CM et al. "The Effect of Exercise Therapy Combined with Psychological Therapy on Physical Activity and Quality of Life in Patients with Painful Diabetic Neuropathy: A Systematic Review." Scandinavian journal of pain, vol. 19, no. 3, 2019, pp. 433-439.

[89] Kluding, Patricia $M$ et al. "The Effect of Exercise on Neuropathic Symptoms, Nerve Function, and Cutaneous Innervation in People with Diabetic Peripheral Neuropathy." Journal of Diabetes and its Complications, vol. 26, no. 5, 2012, pp. 424-429. 
[90] Green, Simon et al. "Cardiovascular Control During Exercise in Type 2 Diabetes Mellitus." Journal of diabetes research, vol. 2015, 2015.

[91] Gu, Yu et al. "Aerobic Exercise Training May Improve Nerve Function in Type 2 Diabetes and Pre - Diabetes: A Systematic Review." Diabetes/metabolism research and reviews, vol. 35, no. 2, 2019, p. e3099.

[92] Jung, Sang Hoon et al. "Aldose Reductase and Advanced Glycation Endproducts Inhibitory Effect of Phyllostachys Nigra." Biological and Pharmaceutical Bulletin, vol. 30, no. 8, 2007, pp. 1569-1572.

[93] Brownlee, M. "Banting Lecture 2004." Diabetes, vol. 54, 2005, pp. 1615-1625.

[94] Singleton, J Robinson et al. "Exercise as Therapy for Diabetic and Prediabetic Neuropathy." Current diabetes reports, vol. 15 , no. 12,2015 , p. 120.

[95] Gilani, Seyed Reza Mousavi and Abdurrashid Khazaei Feizabad. "The Effects of Aerobic Exercise Training on Mental Health and Self-Esteem of Type 2 Diabetes Mellitus Patients." Health psychology research, vol. 7, no. 1, 2019.

[96] McCarthy, Olivia et al. "Resistance Isn't Futile: The Physiological Basis of the Health Effects of Resistance Exercise in Individuals with Type 1 Diabetes." Frontiers in Endocrinology, vol. 10, 2019.

[97] Cooke, Samuel et al. "Effects of Exercise, Cognitive, and Dual-Task Interventions on Cognition in Type 2 Diabetes Mellitus: A Systematic Review and Meta-Analysis." PloS one, vol. 15 , no. 5,2020 , p. e0232958.

[98] Van Tienen, FHJ et al. "Physical Activity Is the Key Determinant of Skeletal Muscle Mitochondrial Function in Type 2 Diabetes." The Journal of Clinical Endocrinology \& Metabolism, vol. 97, no. 9, 2012, pp. 3261-3269.

[99] Phielix, Esther et al. "Exercise Training Increases Mitochondrial Content and Ex Vivo Mitochondrial Function Similarly in Patients with Type 2 Diabetes and in Control Individuals." Diabetologia, vol. 53, no. 8, 2010, pp. 17141721 .

[100] Bloomer, Richard J and Allan H Goldfarb. "Anaerobic Exercise and Oxidative Stress: A Review." Canadian journal of applied physiology, vol. 29, no. 3, 2004, pp. 245-263.

[101] Di Meo, Sergio and Paola Venditti. "Mitochondria in Exercise-Induced Oxidative Stress." Neurosignals, vol. 10, no. 1-2, 2001, pp. 125-140.

[102] Castrogiovanni, Paola and Rosa Imbesi. "Oxidative Stress and Skeletal Muscle in Exercise." Italian journal of anatomy and embryology, vol. 117, no. 2, 2012, p. 107.

[103] Urso, Maria L and Priscilla M Clarkson. "Oxidative Stress, Exercise, and Antioxidant Supplementation." Toxicology, vol. 189, no. 1-2, 2003, pp. 41-54.

[104] Muench, John et al. "Implementing a Team-Based Sbirt Model in Primary Care Clinics." Journal of Substance Use, vol. 20, no. 2,2015 , pp. 106-112.

[105] Ramasamy, Ravichandran et al. "Receptor for Advanced
Glycation Endproducts (Rage): A Formidable Force in the Pathogenesis of the Cardiovascular Complications of Diabetes \& Aging." Current molecular medicine, vol. 7, no. 8, 2007, pp. 699-710.

[106] Magalhães, Pedro $M$ et al. "Involvement of Advanced Glycation End Products in the Pathogenesis of Diabetic Complications: The Protective Role of Regular Physical Activity." European Review of Aging and Physical Activity, vol. 5, no. 1, 2008, pp. 17-29.

[107] Radoi, VALENTIN et al. "Advanced Glycation End Products in Diabetes Mellitus: Mechanism of Action and Focused Treatment." Proceedings of the Romanian Academy, Series B, vol. 1, 2012, pp. 9-19.

[108] Boor, Peter et al. "Regular Moderate Exercise Reduces Advanced Glycation and Ameliorates Early Diabetic Nephropathy in Obese Zucker Rats." Metabolism, vol. 58, no. 11, 2009, pp. 1669-1677.

[109] Sehn, Luiz Fernado Biazus et al. "Effects of Physical Exercise on Cognitive Function of Older Adults with Mild Cognitive Impairment: A Systematic Review and Meta-Analysis." Archives of Gerontology and Geriatrics, 2020, p. 104048.

[110] Wu, Zhi-Jian et al. "The Impact of Interval Training on Cardiorespiratory Fitness, Body Composition, Physical Fitness, and Metabolic Parameters in Older Adults: A Systematic Review and Meta-Analysis." 2020.

[111] Willoughby, DARRYN S and LEMUEL Taylor. "Effects of Sequential Bouts of Resistance Exercise on Androgen Receptor Expression." Medicine and science in sports and exercise, vol. 36, no. 9, 2004, pp. 1499-1506.

[112] Kraemer, William $\mathrm{J}$ et al. "Acute Hormonal Responses to Heavy Resistance Exercise in Younger and Older Men." European journal of applied physiology and occupational physiology, vol. 77, no. 3, 1998, pp. 206-211.

[113] Herbst, Karen L and Shalender Bhasin. "Testosterone Action on Skeletal Muscle." Current Opinion in Clinical Nutrition \& Metabolic Care, vol. 7, no. 3, 2004, pp. 271-277.

[114] Kraemer, William J and Nicholas A Ratamess. "Hormonal Responses and Adaptations to Resistance Exercise and Training." Sports medicine, vol. 35, no. 4, 2005, pp. 339-361.

[115] Baker, Jeremy $\mathrm{R}$ et al. "Effects of Age on Testosterone Responses to Resistance Exercise and Musculoskeletal Variables in Men." Journal of Strength and Conditioning Research, vol. 20, no. 4, 2006, p. 874.

[116] Izquierdo, M et al. "Effects of Strength Training on Muscle Power and Serum Hormones in Middle-Aged and Older Men." Journal of applied physiology, vol. 90, no. 4, 2001, pp. 1497-1507.

[117] Nicklas, BJ et al. "Testosterone, Growth Hormone and Igf-I Responses to Acute and Chronic Resistive Exercise in Men Aged 55-70 Years." International journal of sports medicine, vol. 16 , no. 07,1995 , pp. 445-450.

[118] Hawkins, Vivian N et al. "Effect of Exercise on Serum Sex Hormones in Men: A 12-Month Randomized Clinical Trial." Medicine and science in sports and exercise, vol. 40, no. 2, 2008, p. 223. 